\title{
Hepatic Clostridium Perfringens Abscess Formation after Radiofrequency Ablation Therapy for Hepatocellular Carcinoma: Report of a Rare Case
}

\author{
Ming-Hung Wang Yuan-Hung Kuo Yi-Hao Yen Sheng-Nan Lu \\ Jing-Houng Wang Chien-Hung Chen Chao-Hung Hung \\ Kwong-Ming Kee
}

Division of Hepatogastroenterology, Department of Internal Medicine, Kaohsiung Chang Gung Memorial Hospital, and Chang Gung University College of Medicine, Kaohsiung City, Taiwan

\section{Keywords}

Liver abscess - Radiofrequency ablation · Hepatocellular carcinoma $\cdot$ Clostridium perfringens

\begin{abstract}
Liver abscess formation is one of the major complications following radiofrequency ablation (RFA) in patients with hepatocellular carcinoma (HCC). Clostridium perfringens is a rare but fatal (mortality rate: $70-100 \%$ ) organism that could lead to severe sepsis. We presented a case where a 63-year-old woman with diabetes mellitus, hypertension, chronic hepatitis B-related cirrhosis in Child-Pugh class A and HCC with initial TNM stage II who had undergone 2 sessions of transarterial chemoembolization. RFA was performed for 4 small HCC due to poor effect of previous transarterial chemoembolization. However, all 4 treated tumors developed liver abscesses presenting with septic shock within 1 day. Aspirated abscesses and blood culture both yielded C. perfringens infection. After intensive care, optimal intravenous antibiotic, and abscesses aspiration, the patient recovered successfully. All tumors achieved complete response during the follow-up period without local recurrence. The clinical presentations and risk factors of $C$. perfringens-related liver abscess after RFA will be discussed in this manuscript.
\end{abstract}




\section{Introduction}

Hepatocellular carcinoma (HCC) has the sixth highest prevalence of all-cause cancer and is the fourth highest cause of cancer-related death worldwide. Asia is the region of high prevalence rate with $>50 \%$ of newly diagnosed cases occurring in East Asia [1,2]. Radiofrequency ablation (RFA) is one of the curative treatments of early stage HCC, as well as tumor resection and liver transplantation. Several common complications of the RFA procedure include intra-abdominal bleeding, infection, biliary tract trauma, puncture site tumor seeding, visceral damaging or even liver failure, [3] with post-procedural infections being commonly encountered such as liver abscess formation ranging from 0.3 to $1.7 \%$, puncture wound infection and sepsis.

The common organisms of the liver abscess formation reported previously were Enterococcus, E. coli, Bacteroides fragilis, E. faecalis, and Klebsiella pneumonia [3]. Usual clinical presentations of the complication include fever, chills, right upper quadrant abdominal pain, and general malaise [4]. This case report is focused at intra-tumoral liver abscess formation after RFA due to severe Clostridium perfringens infection, which has rarely been documented previously [5-8].

\section{Case Presentation}

A 63-year-old female patient presented as a case of chronic hepatitis B-related cirrhosis in Child-Pugh class A status for over 10 years and received long-term oral antiviral treatment with entecavir $0.5 \mathrm{mg}$ per day with undetectable HBV DNA and normal ALT levels during follow-up period. Her medical history included type II Diabetic mellitus (DM) under oral antidiabetic agent control and hypertension under medical treatment, and her daily activity was totally independent. In addition, she had been diagnosed as HCC for 2 years. Before admission this time, her initial HCC staging was AJCC/TNM stage II and BCLC stage B, and she underwent 2 sessions of transarterial chemoembolization (TACE).

The follow-up contrast-enhanced CT in December 17,2016, showed 2 tumors with typical image of HCC at segment $5(2.4$ and $1.1 \mathrm{~cm})$ and 2 new poorly enhanced nodules in segments $2(1.7 \mathrm{~cm})$ and $8(2.8 \mathrm{~cm})$ with suspicion of recurrent HCC. There was no extrahepatic metastasis or major vascular invasion. Due to poor treatment response to previous TACE, it was decided to treat the 4 tumors with RFA after discussion with the patient. She received ultrasound-guided RFA with $3 \mathrm{~cm}$ length internal cool tip system (Medtronic, Minneapolis, MN, USA) for the 4 liver tumors under general anesthesia in this treatment course.

Unfortunately, she suffered from acute onset of fever, chills, dyspnea, and abdominal pain $6 \mathrm{~h}$ after RFA. Physical examination showed body temperature had increased to $39.6^{\circ} \mathrm{C}$, heart rate had increased up to 130 beats per min, and blood pressure was 90/46 $\mathrm{mm} \mathrm{Hg}$. Laboratory studies were as follows: WBC $24,600 / \mu \mathrm{L}$ with neutrophil predominant (85\%), hyperlactatemia: $65.4 \mathrm{mg} / \mathrm{dL}, \mathrm{CRP}: 51 \mathrm{mg} / \mathrm{L}, \mathrm{AST}: 239 \mathrm{U} / \mathrm{L}$, ALT: $80 \mathrm{U} / \mathrm{L}$, total bilirubin: $1.1 \mathrm{mg} / \mathrm{dL}$,

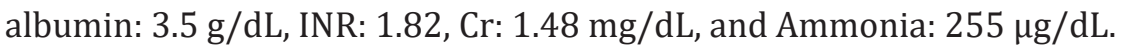

Abdominal ultrasound was performed and showed hyperechoic change with air formation in the treated tumors, while contrast-enhanced CT-indicated necrotic changes with air component in all 4 treated tumors with favorable abscess formation (Fig. 1, 2). As a result, intra-tumoral abscess formation associated with septic shock was impressed, so the patient was transferred to the intensive care unit emergently, with oxygen supplement, aggressive fluid resuscitation, and empirical antibiotic with intravenous Flomoxef prescribed. Ultrasound-guided aspiration to the lesions yielded some pus discharge, and then the pus in lesions was aspirated adequately and sent for culture. Packed red blood cell and fresh frozen plasma were transfused for anemia and coagulopathy correction.

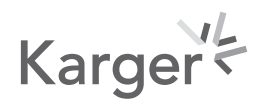




\section{Case Reports in Oncology}

Fig. 1. (Transverse view) show contrast-enhanced CT at the day after RFA procedure and the results show necrotic changes with air component in all 4 treated tumors (white arrow). RFA, radiofrequency ablation.

Fig. 2. (Coronal view) show contrast-enhanced CT at the day after RFA procedure and the results show necrotic changes with air component in all 4 treated tumors (white arrow). RFA, radiofrequency ablation.
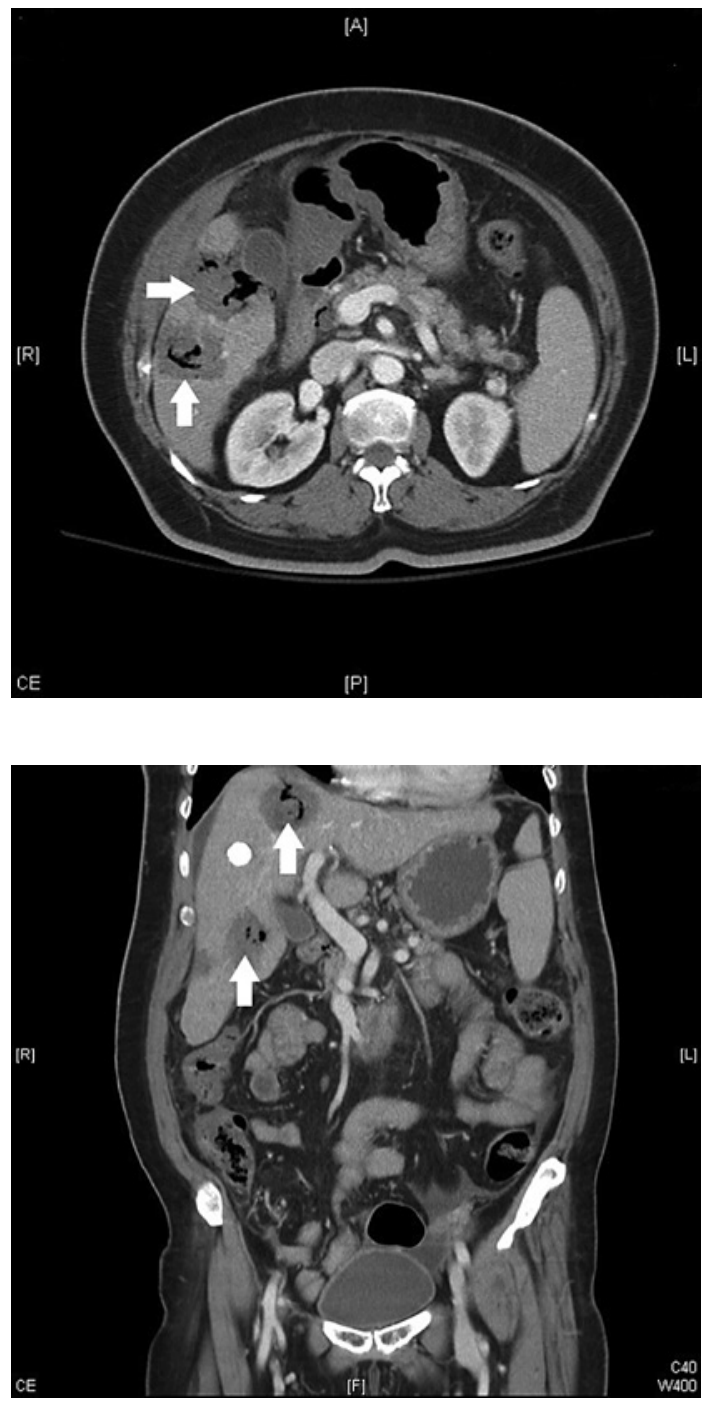

Three days later, the liver abscess and the blood culture both revealed $C$. perfringens bacterial infection, and the drug sensitivity test showed the bacteria was susceptible to Flomoxef. Several sessions of ultrasound-guided abscesses aspiration were performed and a course of antibiotics was completed, with the infection being brought gradually under control after the intensive treatment. The patient was then discharged with stable condition 30 days after the RFA and is still alive after 3 years of follow-up treatment, with follow-up contrastenhanced CT or MRI every 3 months showing complete ablation of the treated tumors and no local tumor recurrence.

\section{Discussion}

RFA is an effective tool for patients in BCLC stage A with tumor number $<3$ or tumor size $<3 \mathrm{~cm}$, whose general condition is not feasible for HCC tumor resection nor liver transplantation. Regarding this patient, she had 4 HCC nodules with the largest tumor size being $2.8 \mathrm{~cm}$ and was in BCLC stage B to which TACE recommends in the practice guidelines. In BCLC stage $B$, there are variations of tumor size $(>3 \sim 10 \mathrm{~cm})$, tumor number $(4 \sim 20)$, and liver function 
(Child-Pugh score: 5-9). By the Kinki criteria of Japan, BCLC stage B can be divided into 3 different stages from B1 to B3 and survival rates reflect significant differences among the 3 subgroups [9]. For patients in BCLC B1 status, when tumor numbers are $\leq 7$, they have better survival benefit and curative potential. Because the previous response of TACE was insufficient for the patient, RFA treatment was recommended and she eventually achieved complete ablation and recovery during the follow-up. A previous study showed HCC patients who developed liver abscesses after treatment with TACE benefited from partial or complete response and were associated with markedly decreased serum AFP levels [10], so this indicates that liver abscess formation might lead to necrosis or devascularization of the liver tumor.

It has been estimated that the complication rate of RFA treatment is about 2-5.7\% [3], with complications including intra-abdominal hemorrhage, hepatic injuries with bile duct injury, liver abscess, puncture site infection, puncture tract seeding, and liver infarction. Others include extrahepatic organ injury following pleural effusions, hemothorax, pneumothorax, skin injuries, and gastrointestinal injuries $[3,11]$.

The incidence of liver abscess was calculated as $0.106-0.196 \%$ of total RFA complications, with common symptoms of abscess being fever, chills, nausea, vomiting, right upper quadrant abdominal pain, and jaundice [4]. Child-Pugh class B and C cirrhosis, biliary tract disease (bilio-enteric anastomosis and endoscopic papillotomy), DM, and porta hepatis tumors are the 4 significant risk factors for abscess formation after RFA [12].

A previous study showed that patients suffering from liver abscess with DM had significantly lower 6-month survival rate if compared with patients without DM (81.97 vs. 92.17\%) [13]. The possible mechanisms of hyperglycemia that influence the prognosis of liver abscess might enhance bacterial growth, inhibit the cellular immune response, and weaken the bactericidal strength of the hematological system $[12,13]$.

Local ablation of the tumor around porta hepatis within $1 \mathrm{~cm}$ of the main biliary tract system may result in bilomas, which connect to the main bile duct and cause retrograde infection [12]. However, most of the bilomas tend to decrease in size and gradually resolve spontaneously [14]. The mortality rate of liver abscess related to biliary origin is $11-15 \%$; on the other hand, the mortality rate of liver abscess of nonbiliary origin is about $3-6 \%$, and in those patients with previous biliary tract disease, using prophylactic antibiotics might lead to much lower incidence rate of developing post-procedural liver abscess than those who did not receive prophylactic antibiotics (3 vs. 17.3\%) [12].

C. perfringens is a Gram-positive, spore-forming, anaerobic, rod-shaped bacterium $[15,16]$. It is commonly distributed in the environment in soils, foods, and the gastrointestinal tract and in the normal flora of the female genital tract [17]. C. perfringens infection most commonly causes food-poisoning and anaerobic cellulitis, and it might also cause severe infection with gas gangrene, septic shock, myositis, and hemolysis [18]. Few studies have discussed the emergence of $C$. perfringens-induced liver abscess associated with post-RFA and microwave ablation treatment [5-8], as it has been a rare organism of liver abscess originating from local ablation treatment. Two papers have mentioned malignancies originating from gastric and colonic regions with metastasis to liver $[5,8]$, with one of the articles reporting microwave ablation of the liver metastasis had been performed [8], while one of the patients developed $C$. perfringens-related liver abscess and expired from cardiopulmonary failure 16 days after the procedure [5].

Our patient was infected with $C$. perfringens-related intra-tumoral abscess with all 4 ablated HCC, being the first to be reported in the literature. The source of infection was not completely explored in this patient, although possible causes of liver abscess related to $C$. perfringens were an abnormal biliary system with retrograde infection from the gastrointestinal tract or retained iodized oil from the previous TACE [7]. C. perfringens infection can lead

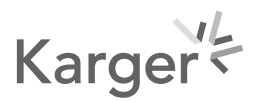


to severe sepsis and hemolysis with high mortality rate (70-100\%). The severity of infection also depends on the underlying condition of the patients [18], where patients with DM, malignancies, biliary tract disease, Child-Pugh class B and C cirrhosis, and obstetrical infection may have much more serious states of infection.

The clinical presentations of our patient included fever, chills, dyspnea, abdominal pain, and septic shock that progressed rapidly. Liver abscess formation and sepsis in an exceedingly early stage were diagnosed and controlled with intensive care, adequate aspiration of the abscess, and appropriate administration of broad-spectrum empirical antibiotics.

\section{Conclusion}

In conclusion, liver abscess with C. perfringens infection related to the post-RFA procedure is a rare but possibly fatal complication; therefore, early detection of sepsis and the appropriate treatment for liver abscess formation with $C$. perfringens infection after the RFA procedure might be the best way to deal with this uncommon but severe complication. If patients have potentially high risk of complications with systemic disease including ChildPugh class B and C cirrhosis, biliary tract disease, DM, porta hepatis tumors, and previous TACE procedure, clinicians should always keep in mind that administration of prophylactic antibiotics might be indicated in such cases.

\section{Statement of Ethics}

The written informed consent was obtained from the patient for publication of this case report and any accompanying images.

\section{Conflict of Interest Statement}

The authors have no conflicts of interest to declare.

\section{Funding Sources}

No funding was received for this work.

\section{Author Contributions}

Conceived and designed the analysis: Kwong-Ming Kee, Yuan-Hung Kuo, Ming-Hung Wang, Jing-Houng Wang, and Chien-Hung Chen. Collected the data: Ming-Hung Wang, KwongMing Kee, contributed data or analysis tools: Kwong-Ming Kee, Yuan-Hung Kuo, Sheng-Nan Lu, Jing-Houng Wang, Chien-Hung Chen, Chao-Hung Hung, and Yi-Hao Yen, wrote the paper: Ming-Hung Wang and Kwong-Ming Kee.

\section{Karger'}




\section{References}

1 Sharma A, Mukewar S, Mara KC, Dierkhising RA, Kamath PS, Cummins N. Epidemiologic factors, clinical presentation, causes, and outcomes of liver abscess: a 35-year Olmsted County Study. Mayo Clin Proc Innov Qual Outcomes. 2018;2(1):16-25.

2 Singal AG, Lampertico P, Nahon P. Epidemiology and surveillance for hepatocellular carcinoma: new trends. J Hepatol. 2020;72(2):250-61.

3 Fonseca AZ, Santin S, Gomes LG, Waisberg J, Ribeiro MA Jr. Complications of radiofrequency ablation of hepatic tumors: frequency and risk factors. World J Hepatol. 2014;6(3):107-13.

4 Serraino C, Elia C, Bracco C, Rinaldi G, Pomero F, Silvestri A, et al. Characteristics and management of pyogenic liver abscess: a European experience. Medicine. 2018;97(19):e0628.

5 Kvitting JP, Sandström P, Thorelius L, Kullman E, Borch K, Svanvik J. Radiofrequency ablation of a liver metastasis complicated by extensive liver necrosis and sepsis caused by gas gangrene. Surgery. 2006;139(1):123-5.

6 Yoshikawa T, Ohana M, Fukuda A. High fever after radiofrequency ablation of hepatocellular carcinoma. Gastroenterology. 2018;155(2):e3-4.

7 Choi D, Lim HK, Kim MJ, Kim SJ, Kim SH, Lee WJ, et al. Liver abscess after percutaneous radiofrequency ablation for hepatocellular carcinomas: frequency and risk factors. AJR Am J Roentgenol. 2005;184(6):1860-7.

8 Kyang LS, Bin Traiki TA, Alzahrani NA, Morris DL. Microwave ablation of liver metastasis complicated by Clostridium perfringens gas-forming pyogenic liver abscess (GPLA) in a patient with past gastrectomy. Int J Surg Case Rep. 2016;27:32-5.

9 Kudo M, Arizumi T, Ueshima K, Sakurai T, Kitano M, Nishida N. Subclassification of BCLC B stage hepatocellular carcinoma and treatment strategies: proposal of modified Bolondi's subclassification (Kinki Criteria). Dig Dis. 2015;33(6):751-8.

10 Jia Z, Tu J, Cao C, Wang W, Zhou W, Ji J, et al. Liver abscess following transarterial chemoembolization for the treatment of hepatocellular carcinoma: a retrospective analysis of 23 cases. J Cancer Res Ther. 2018;14(Suppl): S628-s33.

11 Maeda M, Saeki I, Sakaida I, Aikata H, Araki Y, Ogawa C, et al. Complications after radiofrequency ablation for hepatocellular carcinoma: a Multicenter Study Involving 9,411 Japanese patients. Liver Cancer. 2020;9(1): $50-62$.

12 Su XF, Li N, Chen XF, Zhang L, Yan M. Incidence and risk factors for liver abscess after thermal ablation of liver neoplasm. Hepat Mon. 2016;16(7):e34588.

13 Du Z, Zhou X, Zhao J, Bi J, Ren Y, Zhang J, et al. Effect of diabetes mellitus on short-term prognosis of 227 pyogenic liver abscess patients after hospitalization. BMC Infect Dis. 2020;20(1):145.

14 Chang IS, Rhim H, Kim SH, Kim YS, Choi D, Park Y, et al. Biloma formation after radiofrequency ablation of hepatocellular carcinoma: incidence, imaging features, and clinical significance. AJR Am J Roentgenol. 2010; 195(5):1131-6.

15 Kiu R, Hall LJ. An update on the human and animal enteric pathogen Clostridium perfringens. Emerg Microbes Infect. 2018;7(1):141.

16 Hassan KA, Elbourne LD, Tetu SG, Melville SB, Rood JI, Paulsen IT. Genomic analyses of Clostridium perfringens isolates from five toxinotypes. Res Microbiol. 2015;166(4):255-63.

17 Sarvari KP, Vasas B, Kiss I, Lazar A, Horvath I, Simon M, et al. Fatal Clostridium perfringens sepsis due to emphysematous gastritis and literature review. Anaerobe. 2016;40:31-4.

18 Rogstad B, Ritland S, Lunde S, Hagen AG. Clostridium perfringens septicemia with massive hemolysis. Infection. 1993;21(1):54-6. 